\title{
Brückenschläge zwischen Musikwissenschaft und Informatik - Vorbemerkung
}

Stefanie Acquavella Rauch, Mainz

Andreas Münzmay, Joachim Veit, Detmold

DOI: $10.25366 / 2020.88$

Zitation: Stefanie Acquavella-Rauch, Andreas Münzmay, Joachim Veit, „Brückenschläge zwischen Musikwissenschaft und Informatik - Vorbemerkung", in: Brückenschläge zwischen Musikwissenschaft und Informatik. Theoretische und praktische Aspekte der Kooperation, in Verbindung mit der Fachgruppe Digitale Musikwissenschaft hrsg. von Stefanie Acquavella-Rauch, Andreas Münzmay und Joachim Veit (= Musikwissenschaft: Aktuelle Perspektiven. Bericht über die Jahrestagung der Gesellschaft für Musikforschung 2019 in Paderborn und Detmold, Bd. 3), Detmold, Musikwissenschaftliches Seminar der Universität Paderborn und der Hochschule für Musik Detmold, 2020, S. XI-XV, DOI: $10.25366 / 2020.88$ 


\section{Brückenschläge zwischen Musikwissenschaft und Informatik - Vorbemerkung}

Im Zeichen des digitalen Wandels, der die Geistes- und Kulturwissenschaften an vielen Stellen betrifft und bewegt, kooperieren auch die Disziplinen Informatik und Musikwissenschaft mit zunehmender Intensität. Sie treten - häufig im Zeichen der Formel ,Digital Humanities' - in einen methodischen Dialog über Fragestellungen etwa im Bereich der Semantik, Modellierung, Kodierung, Annotation und Analyse von Daten, der Automatisierung und des Machine Learning, der Musik- und Medieninformatik, der Visualisierung und Vermittlung von Erkenntnissen sowie der Gestaltung neuer Arbeitsumgebungen. Die Entwicklung verändert potenziell das disziplinäre Selbstverständnis und impliziert somit letztlich auch, dass die in solcher Weise kooperierenden Disziplinen im forschungspolitischen Raum neu zu verorten sind.

Der vorliegende Band enthält insgesamt 22 Beiträge von Kolleg*innen beider Fächer, die über ihre persönlichen Themen, Methoden und Fragestellungen, an denen sie arbeiten, in dieser oder jener Weise in den Dialog der Disziplinen eingetreten sind - sei es auf eher theoretischer oder eher praktischer Ebene, in einer Kombination dieser Ansätze oder auch in vor allem pragmatischer Weise. Auf der Grundlage solcher Erfahrungen zeigen die Beiträge damit unterschiedliche Perspektiven und Facetten auf. Als Referate, Blitzvorträge (Lightning Talks) und Poster wurden sie im Rahmen der beiden Jahrestagungen der Gesellschaft für Musikforschung in Osnabrück 2018 sowie in Paderborn 2019 in verschiedenen Formaten vorgestellt und diskutiert.

Die Texte von Elias Berner, Matthias Pasdzierny, Matej Santi, Oleksii Sapov und Matthias Tischer gehen auf das im Jahr 2018 von Andreas Münzmay mit Benjamin Bohl, Dominik Leipold, Frederic von Vlahovits und Barbara Wiermann für die GfM-Fachgruppe Digitale Musikwissenschaft organisierte Symposium Born-digital: Digitales Material als Herausforderung für die Musikwissenschaft zurück. Ausgangspunkt dieses Fachgruppensymposiums war die Beobachtung, dass die schier unübersehbare Fülle digitalen Materials, das überall dort entsteht, wo Musik in digitalen Umgebungen erfunden, gemacht, produziert, publiziert, verteilt, gespeichert, rezipiert und analysiert wird (um nur einige Grundmodi digitaler musikbezogener kultureller Praxen anzudeuten), für die Musikforschung ohne Frage vielfältige Zukunftsaufgaben birgt und sie zur Entwicklung entsprechender digitaler Methoden geradezu herauszufordern scheint. Vorrangiges Ziel dieses zweiten Symposiums der 2017 gegründeten Fachgruppe war es, die verschiedenen Bereiche des Faches über die Fragen, die mit den digitalen Phänomenen, Erzeugnissen, Arbeitsweisen, Instrumenten und Überlieferungen der Musik- wie auch der Forschungskultur der zurückliegenden Jahrzehnte bis heute einhergehen, miteinander ins Gespräch zu bringen. Für analytische, historiografische, ethnografische, organologische, editorische, archivalische und viele andere wissenschaftliche Zugänge zur Musik könnte sich, so die dahinterstehende Überlegung, die ,Digitalität' des Materials als gemeinsamer 
Anknüpfungspunkt und unter Umständen sogar als Chance für ganz neue Beziehungen ${ }^{1}$ und Kooperationen erweisen.

Die Grundfrage, ob und wie ,Digitalität' ein Fach wie die Musikwissenschaft in ihrem disziplinären Zuschnitt verändert und was das speziell auch für das wissenschaftliche Zusammenarbeiten bedeuten könnte, prägte auch das im Folgejahr 2019 im Rahmen der Paderborner Jahrestagung von Andreas Münzmay und Joachim Veit in Kooperation mit der Fachgruppe Digitale Musikwissenschaft veranstaltete Hauptsymposium Brückenschläge - Musikwissenschaft und Informatik im Dialog. Eine zentrale Idee dieser Tagung war es, Vertreter*innen der beiden Disziplinen Informatik und Musikwissenschaft auf Augenhöhe zusammenzubringen. Dabei wurden in besonderer Weise Kolleg*innen der Paderborner Informatik einbezogen, die durch die Zusammenarbeit im Zentrum Musik - Edition - Medien (ZenMEM) mit Fragestellungen der Musikwissenschaft vertraut sind. In vier thematischen Panels zu den Komplexen "Fachhistorische Perspektiven", "Interaktion", "Kodierung/Analyse" sowie "Infrastrukturelle Perspektiven", jeweils bestehend aus zwei bzw. drei Vorträgen, die aus informatischer und musikwissenschaftlicher Sicht als Tandems zusammengestellt wurden, ${ }^{2}$ konnte der digitale Umgang mit dem Forschungsgegenstand Musik aus beiden Blickwinkeln beleuchtet und auch die Frage, welche Forschungsinteressen beide Seiten in einer solchen Verbindung verwirklichen könnten, ausführlich diskutiert werden. Für eine Publikation der Beiträge konnten Miriam Akkermann, Reinhard Keil, Ulrich Konrad, Shintaro Miyazaki, Gudrun Oevel und Christine Siegert gewonnen werden; besonders hervorzuheben ist dabei die große Bereitschaft aller, für die Schriftfassungen auch Themenstränge aus den Diskussionen aufzugreifen und zu reflektieren.

Flankierend zu den Vorträgen bereicherte die im Namen der Fachgruppe Digitale Musikwissenschaft von Stefanie Acquavella-Rauch organisierte Posterpräsentation das Symposium, sodass den theoretischen Überlegungen der Referate gleichsam als Spiegel praktische Überlegungen und Projektbeispiele auf insgesamt 15 Postern unmittelbar gegenüber gestellt waren. ${ }^{3}$ Zwölf dieser Poster wurden von ihren Autor*innen und Präsentator*innen Stefanie Acquavella-Rauch, Agnes Amminger/Franz Kelnreiter, Axel Beer/Martin Bierwisch/Kristina Krämer, Susanne Cox/Richard Sänger, Annette van Dyck-Hemming/Jan Eberhardt/Melanie Wald-Fuhrmann, Daniel Fütterer, Anna Neovesky/Frederic von Vlahovits, Markus Neuwirth/Johannes Hentschel/Martin Rohrmeier, Dennis Ried, Elisabeth Treydte, Agnes Seipelt ausformuliert und konnten in Beitragsform, inklusive des gezeigten Posters selbst, in die vorliegende Publikation aufgenommen werden.

Obwohl die Beiträge also auf insgesamt drei verschiedene Veranstaltungen bzw. Veranstaltungsformen zurückgehen, haben wir uns für die Publikation zu einer übergreifenden Neu-

1 Z. B. im Sinne der von Georgina Born vor zehn Jahren geforderten Relationen: Georgina Born, "For a Relational Musicology: Music and Interdisciplinarity, Beyond the Practice Turn", in: Journal of the Royal Musical Association, 135/2 (2010), S. 205-243, <http://dx.doi.org/10.1080/02690403.2010.506265>.

2 Siehe Programmheft der Jahrestagung 2019, <https://www.muwi-detmold-paderborn.de/fileadmin/muwi/GfM/ Programmheft_GfM2019_online.pdf>, ab S. 82.

3 Siehe Programmheft, ab S. 273. 
gruppierung entschieden, um jenem Aufeinander-angewiesen-Sein und Ineinandergreifen von ,Theorie' und ,Praxis' sowie von ,Materialbeschaffenheit' und ,Methode', das sich gleichsam als ein Gesamtergebnis aus den genannten Veranstaltungen verstehen lässt, Rechnung zu tragen: In der Abteilung "Kollaborationen - Ko-Laboratorien" geht es zunächst um Denkvoraussetzungen, strukturelle Bedingungen und mögliche Vorbilder für erfolgreiches interdisziplinäres Zusammenarbeiten. Die unter "Text/Daten/Prozesse" gruppierten Beiträge befassen sich vor diesem Hintergrund gezielt mit Fällen aus dem editionswissenschaftlichen und musikanalytischen Kontext, das heißt mit den teils grundlegend, teils eher im Pragmatischen veränderten Konzeptionen in Bezug auf musikalische Werk- bzw. Notentexte und deren Strukturierung und Prozessierung als Datenkorpora. Demgegenüber rückt die Abteilung „Digital(isiert)e Materialitäten" musikalische und epistemologische Praxen ins Zentrum, die a priori mit digitalen Technologien und Medien verbunden sind. In einem weiten kultur- und medienhistorischen Bogen nehmen die hier versammelten Beiträge das Spannungsfeld zwischen Musik, Medialem und dem digitalen Raum bis hin zum allfälligen Digitalitätsbegriff selbst kritisch ins Visier. Mit den Möglichkeiten und Chancen einer dezidiert netzbasierten (auf die Idee technischer Vernetzung gegründeten, zugleich theoretisch als Netzwerke vorgestellten) Historiographie und Geschichtsvermittlung, aber auch mit den Dynamiken von Geschichtsbetrachtungen in sozialen Netzwerken, befassen sich schließlich die fünf Beiträge unter "Musikgeschichte(n) im Netz".

Der "Austausch über Arbeits- und Publikationsmöglichkeiten innerhalb der Disziplin ebenso wie Forschungsansätze und methodische Überlegungen, die einerseits Ursache und andererseits Folge vielfältiger, mit der Digitalität zusammenhängender Veränderungen sind", aber auch die "Reflexion von Konsequenzen ,der' Digitalisierung" sind - so benannte es der 2017 bei der Jahrestagung in Kassel eingebrachte Antrag zur Einrichtung der Fachgruppe - Kernanliegen der der Fachgruppe Digitale Musikwissenschaft im Allgemeinen und aller am vorliegenden Band Beteiligten im Besonderen. Forschungsbemühungen, die sich als ,Digital Humanities' begreifen, sind womöglich von Vornherein stets Brückenschläge, oder sie sind zumindest auf solche angewiesen. Nicht allein die angewandte, sondern gerade auch die wissenschaftliche Informatik ist, so unsere Überzeugung, ein entscheidender Gesprächspartner, und umso mehr hoffen wir, dass der vorliegende Band auf seine Weise dazu beitragen kann, das Verhältnis zwischen Informatik und Musikwissenschaft zu pflegen, es auszuloten und zu beschreiben und zum Gegenstand gemeinsamer wissenschaftlicher Überlegungen zu machen.

Allen voran den Autor*innen, aber auch allen Diskutant*innen nach den Vorträgen und an den Posterstellwänden, möchten wir daher an dieser Stelle sehr dafür danken, dass sie sich auf solche Überlegungen eingelassen und ihre Erfahrungen als Bausteine, Widerlager, Pfeiler und Geländer zur Verfügung gestellt haben! Ebenso gedankt sei den Veranstalter*innen der beiden Jahrestagungen der Gesellschaft für Musikforschung in Osnabrück und Paderborn/Detmold. Ein spezieller Dank richtet sich an die beiden Herausgeberinnen der die Ergebnisse der Paderborner Jahrestagung zusammenfassenden Reihe Musikwissenschaft: Aktuelle Perspektiven, 
Rebecca Grotjahn und Nina Jaeschke, für die Möglichkeit zu dieser umfassenden Publikation und dem von der SLUB Dresden im Rahmen des FID Musikwissenschaft betreuten OpenAccess-Fachrepositorium musiconn.publish für die Unterstützung bei der Veröffentlichung.

Mainz und Detmold, im Oktober 2020

Stefanie Acquavella-Rauch, Andreas Münzmay und Joachim Veit

Zitation: Stefanie Acquavella-Rauch, Andreas Münzmay, Joachim Veit, „Brückenschläge zwischen Musikwissenschaft und Informatik - Vorbemerkung", in: Brückenschläge zwischen Musikwissenschaft und Informatik. Theoretische und praktische Aspekte der Kooperation, in Verbindung mit der Fachgruppe Digitale Musikwissenschaft hrsg. von Stefanie Acquavella-Rauch, Andreas Münzmay und Joachim Veit (= Musikwissenschaft: Aktuelle Perspektiven. Bericht über die Jahrestagung der Gesellschaft für Musikforschung 2019 in Paderborn und Detmold, Bd. 3), Detmold, Musikwissenschaftliches Seminar der Universität Paderborn und der Hochschule für Musik Detmold, 2020, S. XI-XV, DOI: $10.25366 / 2020.88$ 



\section{Brückenschläge zwischen Musikwissenschaft und Informatik}

Theoretische und praktische Aspekte der Kooperation

Herausgegeben von Stefanie Acquavella-Rauch,

Andreas Münzmay und Joachim Veit 
Brückenschläge zwischen Musikwissenschaft und Informatik 


\section{Musikwissenschaft: Aktuelle Perspektiven}

Bericht über die Jahrestagung der Gesellschaft für Musikforschung 2019 in Paderborn und Detmold

Herausgegeben von Rebecca Grotjahn und Nina Jaeschke

Band 3 


\section{Brückenschläge zwischen Musikwissenschaft und Informatik}

Theoretische und praktische Aspekte der Kooperation

Beiträge der Symposien zur Digitalen Musikwissenschaft

Osnabrück 2018 und Paderborn 2019

im Rahmen der Jahrestagungen der Gesellschaft für Musikforschung

In Verbindung mit der Fachgruppe Digitale Musikwissenschaft herausgegeben von

Stefanie Acquavella-Rauch, Andreas Münzmay und Joachim Veit

Detmold: Musikwissenschaftliches Seminar der Universität Paderborn und der Hochschule für Musik Detmold 2020 
DOI: $10.25366 / 2020.87$

Online-Version verfügbar unter der Lizenz: Urheberrecht 1.0, $<$ https://rightsstatements.org/page/InC/1.0/?language =de >

Bibliografische Information der Deutschen Nationalbibliothek

Die Deutsche Nationalbibliothek verzeichnet diese Publikation in der Deutschen Nationalbibliografie; detaillierte bibliografische Daten sind im Internet über http://dnb.d-nb.de abrufbar.

\section{Impressum}

Redaktion: Stefanie Acquavella-Rauch, Andreas Münzmay und Joachim Veit Satz: Nina Jaeschke und Joachim Veit

(C) Musikwissenschaftliches Seminar der Universität Paderborn und der Hochschule für Musik Detmold 2020 


\section{INHALT}

Rebecca Grotjahn, Nina Jaeschke

Vorwort zu Band 1-3

IX

Stefanie Acquavella-Rauch, Andreas Münzmay, Joachim Veit

Brückenschläge zwischen Musikwissenschaft und Informatik - Vorbemerkung

$\mathrm{XI}$

\section{KOLLABORATIONEN - KO-LABORATORIEN}

\section{Reinhard Keil}

Der Computer als Denkzeug für hermeneutische Arbeit

\section{Ulrich Konrad}

Philologie und Digitalität. Perspektiven für die Musikwissenschaft im Kontext fächerübergreifender Institutionen

\section{Gudrun Oevel}

Infrastruktureinrichtungen in Forschungsprojekten - Spagat oder Chance?

\section{Dennis Ried}

Erhebung, Transformation und Präsentation digitaler Forschungsdaten

Anna Neovesky, Frederic von Vlahovits

IncipitSearch - Leitfaden zur Zusammenarbeit

Elisabeth Treydte

Clara Schumann \#digital. 40 Jahre Archiv Frau und Musik und der Start in die Digitalisierung

\section{TEXT/DATEN/PROZESSE}

\section{Christine Siegert}

Komponisten-Gesamtausgaben im digitalen Zeitalter: Perspektiven

und Reflexionen am Beispiel Ludwig van Beethovens

Markus Neuwirth, Johannes Hentschel, Martin Rohrmeier

Perspectives of Musical Corpus Studies: The Annotated Mozart Sonatas

Agnes Amminger, Franz Kelnreiter

Leopold Mozarts „Gründliche Violinschule". Zur Textcodierung und -präsentation einer digitalen Edition

\section{Oleksii Sapov}

Algorithmische Automatisierung komplexer Notationsregeln in MEI-XML am Beispiel von Versetzungszeichen 


\section{Susanne Cox, Richard Sänger}

Digitale Fassungsvergleiche am Beispiel von Beethovens Eigenbearbeitungen

Agnes Seipelt

Digitale Edition und Harmonische Analyse mit MEI von Anton Bruckners

Studienbuch

Stefanie Acquavella-Rauch

Musikalische Schaffensprozesse 2.0 - Inkorporation audiovisueller Medien

der populären Musik in Methoden der digitalen Edition

\section{DIGITAL(ISIERT)E MATERIALITÄTEN}

\section{Miriam Akkermann}

(Musik)Instrument (im) Computer

\section{Daniel Fütterer}

Herausforderungen bei der Kodierung von Paratext am Beispiel Neuer Musik mit Live-Elektronik

\section{Matthias Pasdzierny}

How much is the glitch? Das digitale Paradigma als Herausforderung

und Chance für die historische Musikwissenschaft

\section{Shintaro Miyazaki}

Musik für Maschinen?! - Wo sich die Wissenschaft der Medien, des Computers und der Musik treffen und wie sie zusammenarbeiten könnten

\section{MUSIKGESCHICHTE(N) IM NETZ}

\section{Matthias Tischer}

Musikgeschichte der DDR: Ein Pilotprojekt zur digitalen Musikvermittlung

Annette van Dyck-Hemming, Jan Eberhardt, Melanie Wald-Fuhrmann

Ansätze zur Analyse historischer Netzwerke mit Neo4j® - Aus der Projekt-Werkstatt der Datenbank zur Fachgeschichte der Musikwissenschaft

Axel Beer, Martin Bierwisch, Kristina Krämer

Das MMM2 - Ein regionalgeschichtliches Onlinelexikon der Arbeitsgemeinschaft für mittelrheinische Musikgeschichte

\section{Matej Santi}

Was erzählt Fritz Kreislers Geige?

\section{Elias Berner}

Alle Menschen werden Brüder?! Ein historisches Dokument aus dem

Nationalsozialismus in den sozialen Medien 
Gabriele Buschmeier in memoriam 